\title{
VIBRATION OF CRANE RADIUS CHANGE SYSTEM WITH INTERNAL DAMPING
}

\author{
Wojciech Sochacki ${ }^{1}$, Marta Bold ${ }^{2}$ \\ Institute of Mechanics and Fundamentals of Machinery Design \\ Czestochowa University of Technology, Czestochowa, Poland \\ ${ }^{I}$ sochacki@imipkm.pcz.pl, ${ }^{2}$ bold@imipkm.pcz.pl
}

\begin{abstract}
In the present work a problem pertaining to the damped lateral vibrations of the truck crane radius change system with the developed hydraulic cylinder model that changes the radius has been formulated and solved. In the adopted model the vibration energy dissipation derives from the internal damping of the viscoelastic material (the Kelvin-Voigt rheological model) of beams that model the system. Damped vibration frequency and the vibration amplitude decay level have been calculated. Changes of the eigenvalues of system vibrations with the damping ratio change and the change of the system geometry with different loads observed on it have been presented.
\end{abstract}

Keywords: truck crane, vibration damping, eigenvalues

\section{Introduction}

Research on the vibrations of the truck crane radius change system has been the subject of many authors. Comprehensive literature pertaining to vibrations of truck cranes and their elements has been included in monographs $[1,2]$. Studies on free vibrations of the crane radius change system are presented in the work [3]. A similar study has been the subject of the work [4], in which an analysis of free and parametric vibrations of the radius change system has been conducted. The manner of modeling the hydraulic cylinder that changes the radius in the abovementioned works was simplified and the impact of damping has not been included in the systems.

The present work considers damped vibrations of the DST0285 truck crane radius change system. The vibration energy dissipation during the radius change arises due to the internal damping of the viscoelastic material of the system. Similar solutions are presented in works [5, 6]. In the work [5] an impact of small internal and outer damping on the stability of non-conservative beam systems has been revealed. Studies included in the work [6] depict the influence of internal damping on the vibrations of the cantilever beam with mass attached at the free end of the beam.

\section{Physical and mathematical model of the system}

The physical model of the system is presented in Figure 1. The radius change (boom inclination angle) is performed by means of a hydraulic cylinder mounted 
on the rotary frame of the boom. The telescopic boom is composed of three steel units, where one of them is a stationary unit whereas other two are extension units, which are extended simultaneously with the hydraulic cylinder of the cylinder output stroke and the mechanical linear system cooperating with it.

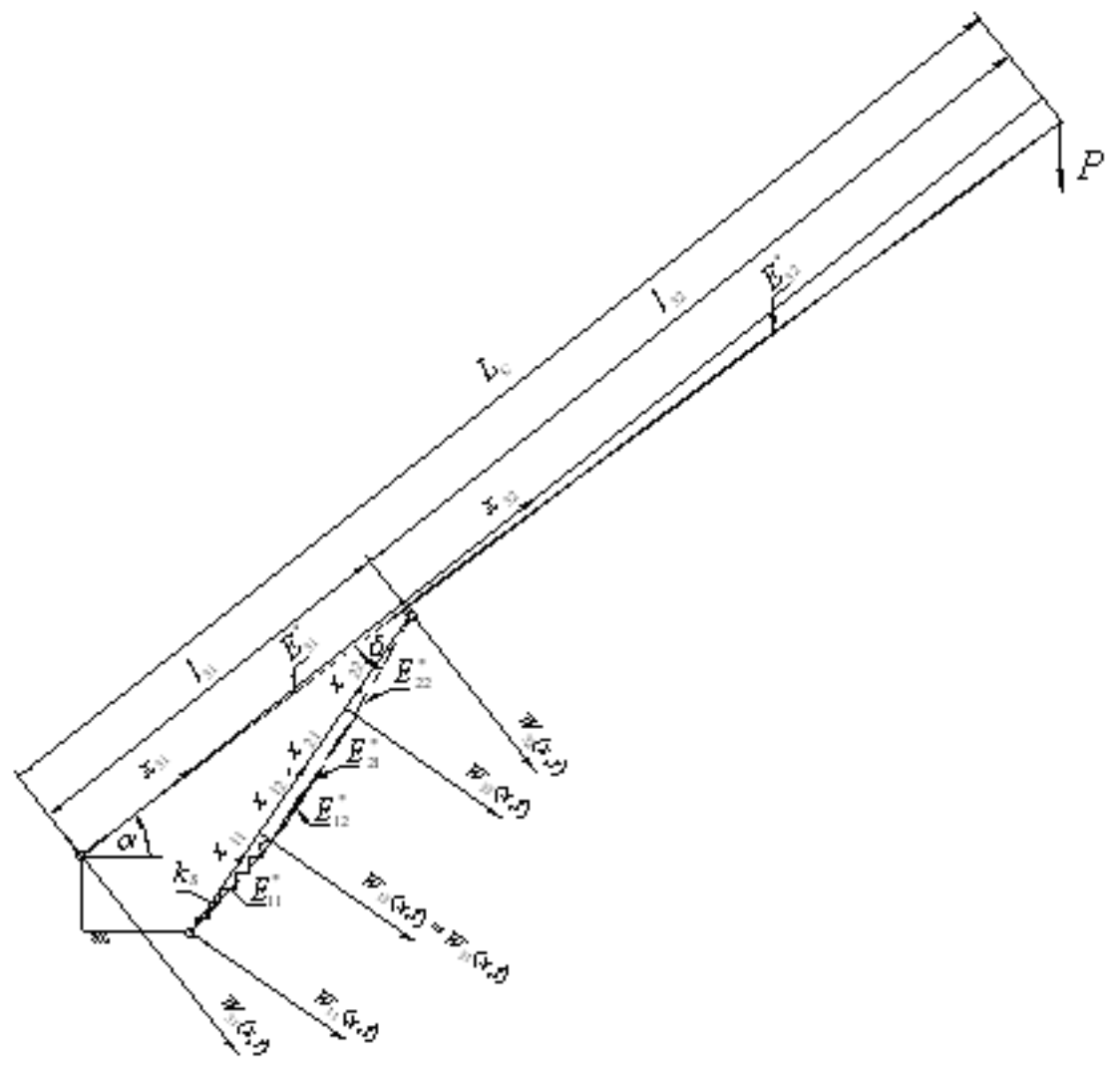

Fig. 1. The physical model of the crane radius change system

Viscoelastic material has been characterized by the Young's modulus $E$ and the viscosity coefficient $E^{*}$ of both the hydraulic cylinder material and boom material.

Equations of the beam movement modeling the radius change system are as follows:

$E_{m n} J_{m n} \frac{\partial^{4} W_{m n}(x, t)}{\partial x^{4}}+E_{m n}^{*} J_{m n} \frac{\partial^{5} W_{m n}(x, t)}{\partial x^{4} \partial t}+P_{m n} \frac{\partial^{2} W_{m n}(x, t)}{\partial x^{2}}+\rho_{m n} A_{m n} \frac{\partial^{2} W_{m n}(x, t)}{\partial t^{2}}=0$

where:

$W_{m n}(x, t)$ - the lateral displacement of beams,

$E_{m n} \quad$ - Young's moduli for particular beams,

$E_{m n}^{*} \quad$ - the viscosity coefficient of the material of particular beams, 
$A_{m n}$ - beam section areas,

$J_{m n}$ - the moment of inertia for beam sections,

$\rho_{m n}$ - the density of the beam material,

$P_{m n}$ - longitudinal forces in beams modeling the boom and the hydraulic cylinder of radius change $\left(P_{12}=0\right)$,

$m=1,2,3 ; n=1,2$

$x$ - space coordinate,

$t$ - time.

Solutions of equations (1) are in the form:

$$
W_{m n}(x, t)=w_{m n}(x) e^{i \omega^{*} t}
$$

where: $\omega^{*}$ - the complex eigenvalue of the system, $i=\sqrt{-1}$.

Substitution of (2) into (1) leads to:

$$
w_{m n}^{I V}(x)+\beta_{m n}^{2} w_{m n}^{I I}(x)-\gamma_{m n} w_{m n}(x)=0
$$

where:

$$
\gamma_{m n}=\frac{\rho_{m n} A_{m n} \omega^{* 2}}{\left(E_{m n}+i E_{m n}^{*} \omega^{*}\right) J_{m n}}, \quad \beta_{m n}=\sqrt{\frac{P_{m n}}{\left(E_{m n}+i E_{m n}^{*} \omega^{*}\right) J_{m n}}}, \beta_{12}=0
$$

Geometric boundary conditions and continuity conditions are as shown below:

$$
\begin{aligned}
& w_{11}(0)=0, \quad w_{11}\left(l_{11}\right)=w_{12}(0), \quad w_{11}^{I}\left(l_{11}\right)=w_{12}^{I}(0), \\
& w_{12}(0)=w_{21}(0), \quad w_{12}\left(l_{12}\right)=w_{21}\left(l_{21}\right), \quad w_{21}\left(l_{21}\right)=w_{22}(0), \\
& w_{21}^{I}\left(l_{21}\right)=w_{22}^{I}(0), \quad w_{22}\left(l_{22}\right)=w_{31}\left(l_{31}\right) \cos \delta, \quad w_{31}(0)=0, \\
& w_{31}\left(l_{31}\right)=w_{32}(0), \quad w_{31}^{I}\left(l_{31}\right)=w_{32}^{I}(0)
\end{aligned}
$$

Natural boundary conditions of the studied system are as follows:

$$
\begin{aligned}
& \left(E_{11}+i E_{11}^{*} \omega^{*}\right) J_{11} w_{11}^{I I}(0)=0,\left(E_{12}+i E_{12}^{*} \omega^{*}\right) J_{12} w_{12}^{I I}\left(l_{12}\right)=0, \\
& \left(E_{12}+i E_{12}^{*} \omega^{*}\right) J_{12} w_{12}^{I I}(0)=\left(E_{11}+i E_{11}^{*} \omega^{*}\right) J_{11} w_{11}^{I I}\left(l_{11}\right),\left(E_{21}+i E_{21}^{*} \omega^{*}\right) J_{21} w_{21}^{I I}(0)=0, \\
& \left(E_{11}+i E_{11}^{*} \omega^{*}\right) J_{11} w_{11}^{I I I}\left(l_{11}\right)+P_{11} w_{11}^{I}\left(l_{11}\right)-\left(E_{12}+i E_{12}^{*} \omega^{*}\right) J_{12} w_{12}^{I I I}\left(l_{12}\right)+ \\
& +\left(E_{21}+i E_{21}^{*} \omega^{*}\right) J_{21} w_{21}^{I I I}(0)+P_{21} w_{21}^{I}(0)=0, \\
& \left(E_{12}+i E_{12}^{*} \omega^{*}\right) J_{12} w_{12}^{I I I}\left(l_{12}\right)+\left(E_{21}+i E_{21}^{*} \omega^{*}\right) J_{21} w_{21}^{I I I}\left(l_{21}\right)+ \\
& -\left(E_{22}+i E_{22}^{*} \omega^{*}\right) J_{22} w_{22}^{I I I}(0)=0,\left(E_{31}+i E_{31}^{*} \omega^{*}\right) J_{31} w_{31}^{I I}(0)=0, \\
& \left(E_{32}+i E_{32}^{*} \omega^{*}\right) J_{32} w_{32}^{I I I}\left(l_{32}\right)+P_{32} w_{32}^{I}\left(l_{32}\right)=0,
\end{aligned}
$$




$$
\begin{aligned}
& \left(E_{22}+i E_{22}^{*} \omega^{*}\right) J_{22} w_{22}^{I I}\left(l_{22}\right)=0,\left(E_{32}+i E_{32}^{*} \omega^{*}\right) J_{32} w_{32}^{I I}\left(l_{32}\right)=0, \\
& \left(E_{22}+i E_{22}^{*} \omega^{*}\right) J_{22} w_{22}^{I I}(0)=\left(E_{21}+i E_{21}^{*} \omega^{*}\right) J_{21} w_{21}^{I I}\left(l_{21}\right), \\
& \left(E_{31}+i E_{31}^{*} \omega^{*}\right) J_{31} w_{31}^{I I}\left(l_{31}\right)=\left(E_{32}+i E_{32}^{*} \omega^{*}\right) J_{32} w_{32}^{I I}(0), \\
& \left(E_{31}+i E_{31}^{*} \omega^{*}\right) J_{31} w_{31}^{I I I}\left(l_{31}\right)-\left(E_{32}+i E_{32}^{*} \omega^{*}\right) J_{32} w_{32}^{I I I}(0)+ \\
& +\left[\left(E_{22}+i E_{22}^{*} \omega\right) J_{22} w_{22}^{I I I}+P_{22} w_{22}^{I}\right] \cos \delta-k_{S} w_{31}\left(l_{31}\right) \sin \delta=0
\end{aligned}
$$

The solution of equations (3) is expressed in the form of functions:

$$
w_{m n}(x)=C_{1 m n} e^{\lambda_{m n} x}+C_{2 m n} e^{-\lambda_{m n} x}+C_{3 m n} e^{i \bar{\lambda}_{m n} x}+C_{4 m n} e^{-i \bar{\lambda}_{m n} x}
$$

where: $\lambda_{m n}=\sqrt{-\frac{\beta_{m n}^{2}}{2}+\sqrt{\frac{\beta_{m n}^{4}}{4}+\gamma_{m n}}}, \bar{\lambda}_{m n}=\sqrt{\frac{\beta_{m n}^{2}}{2}+\sqrt{\frac{\beta_{m n}^{4}}{4}+\gamma_{m n}}}$

By substituting (5) into (4a-b) a homogeneous system of equations was obtained with respect to the unknown constants $C_{k m n}$, which in the matrix form can be written as:

$$
[A]\left(\omega^{*}\right) C=0
$$

where: $A\left(\omega^{*}\right)=\left[a_{p q}\right],(p, q=1,2 . .24), C=\left[C_{k m n}\right]^{\mathrm{T}}, k=1,2-4$.

The system has a nontrivial solution when the matrix determinant of coefficients is equal to zero with constants $C_{k m n}$.

$$
\operatorname{det} A\left(\omega^{*}\right)=0
$$

Finding complex eigenvalues of the matrix $A\left(\omega^{*}\right)$ leads to the determination of damped vibration frequency $\operatorname{Re}\left(\omega^{*}\right)$ and the vibration amplitude decay level $\operatorname{Im}\left(\omega^{*}\right)$ of the considered system.

\section{Numerical calculation results}

Calculations were carried out for the DST0285 truck crane radius change system. In order to present the results of the study the non-dimensional damping parameters $\eta$ for internal damping of the viscoelastic material of beams have been adopted:

$$
\eta=\frac{E^{*}}{c E}
$$

where: $c^{2}=\left(L_{C}+L_{S}\right)^{4} \frac{\sum_{m, n} \rho_{m n} A_{m n}}{\sum_{m, n} E_{m n} J_{m n}}, L_{S}$ - overall length of the hydraulic cylinder. 
Calculation results are depicted in Figures 2-4. Solid lines in figures present the results of the study on the system without load whereas (dashed) lines indicate the results of the study on the system with load equal to $P=50 \mathrm{kN}$. Figures pertain to the study on the influence of internal damping of system elements on its eigenvalues. The dependence of the real part $\operatorname{Re}\left(\omega^{*}\right)$ and imaginary part $\operatorname{Im}\left(\omega^{*}\right)$ of the first two eigenvalues of the radius change system on the internal damping non-dimensional parameter value $\eta$ is presented in Figures 2a,b. Presented results of the study pertain to the boom extended to the length of $L c=15 \mathrm{~m}$ and the angle of its inclination $\alpha=33^{\circ}$.

a)

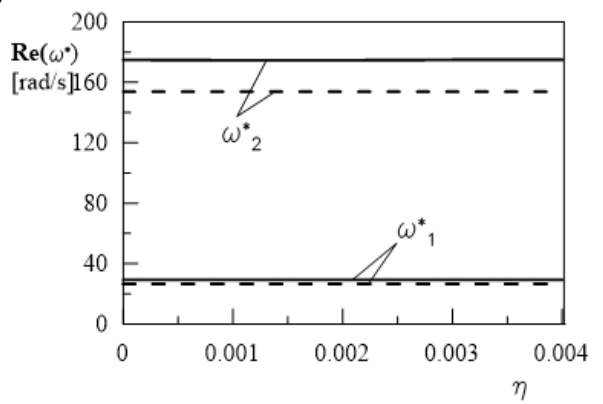

b)

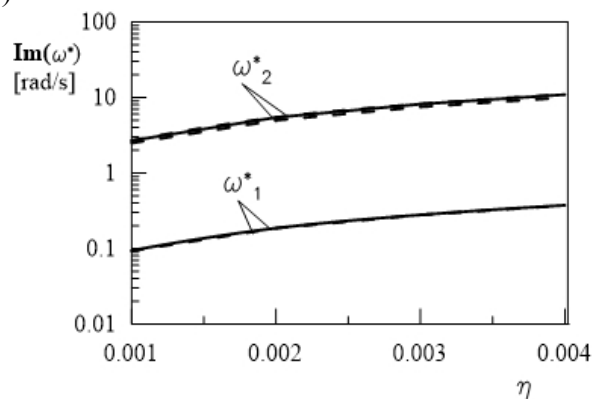

Fig. 2. The dependence of the first two eigenvalues (real parts (a) and imaginary parts (b)) of the crane radius change system on the damping ratio $\eta$

The results of the study on the dependence of the system eigenvalues on the boom inclination angle $\alpha$ are presented in Figure 3a,b. Calculations have been conducted with the damping ratio equal to $\eta=0,002$ and the boom overall length $L c=15 \mathrm{~m}$.

a)

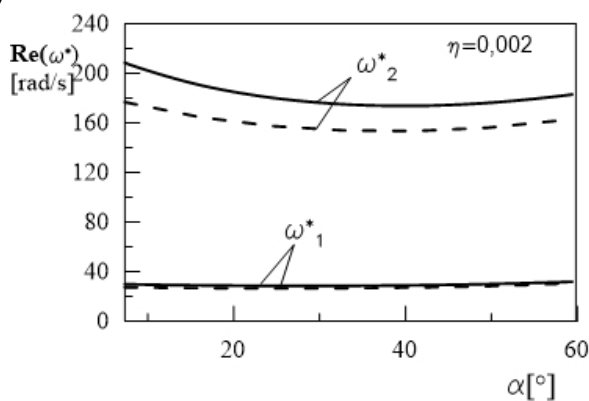

b)

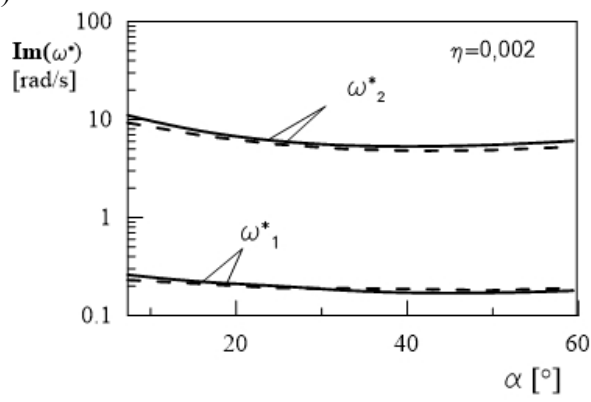

Fig. 3. The dependence of the first two eigenvalues (real parts (a) and imaginary parts (b)) of the crane radius change system on the boom inclination angle $\alpha$

The dependence of the real and imaginary part of the first two eigenvalues of the radius change system on the change of the boom overall length $L_{C}$ (Fig. 4a,b), where the damping ratio is $\eta=0,002$ and the boom inclination angle equals $\alpha=33^{\circ}$, has been indicated in further studies. 
a)

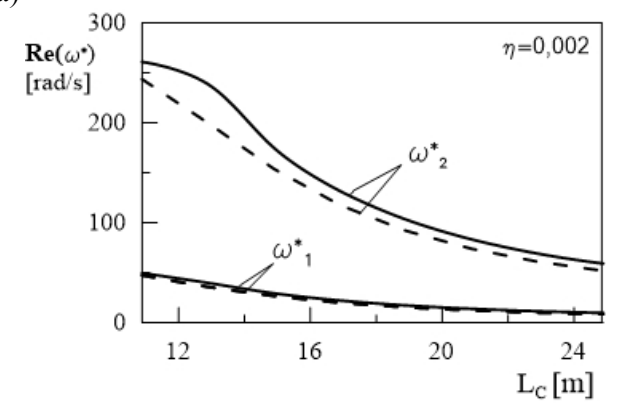

b)

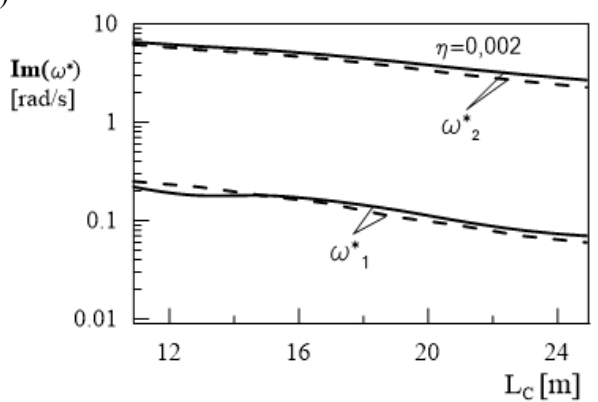

Fig. 4. The dependence of the first two eigenvalues (real parts (a) and imaginary parts (b)) of the crane radius change system on the boom overall length $L_{C}$

The limitation of the research to the analysis of the first two eigenvalues with the geometrical and damping change in the system results from the fact of decisive importance in engineering practice.

\section{Conclusions}

In this work a beam model of the telescopic boom - hydraulic cylinder system developed on the basis of the real system of the DST0285 truck crane has been presented. A study on the influence of the geometry and internal damping of the viscoelastic material of beams modeling the system into the system eigenvalues has been conducted. On the basis of the performed calculations it is possible to state that the inclusion of internal damping into the consideration of the vibrations of the crane radius change system leads only to a small change in its vibration frequency (Fig. 2a) within the entire scope of the study. The analysis of the occurrence of imaginary eigenvalue frequency (Fig. 2b) of the system indicates (as could have been expected) that with the value increase of the damping ratio $\eta$ the rate of the system's vibration amplitude decay is higher.

The change of the boom inclination angle causes a reduction of the second eigenvalue of the system (both $\operatorname{Re}\left(\omega^{*}\right)$ and $\operatorname{Im}\left(\omega^{*}\right)$ ) reaching a minimum at angle $\alpha \approx 41^{\circ}$ and then the eigenvalues slightly increased. The change of the boom inclination angle without essential influence on the first eigenvalue (Fig. 3a,b).

A length change of the boom has significant impact on the lowering of the damped vibration frequency (Fig. 4a) of the first and second eigenvalue. The change of the boom length simultaneously causes substantial lowering of the vibration amplitude decay coefficient of the second eigenvalue and slightly lowers the first eigenvalue.

System load equal to $P=50 \mathrm{kN}$ does not cause significant changes in results obtained with respect to the case without the observance of damping. Load lowers damped vibration frequencies every time without causing substantial changes of the vibration amplitude decay coefficient $\operatorname{Im}\left(\omega^{*}\right)$ of the first and second eigenvalue. 


\section{Acknowledgements}

This research was supported by the Ministry of Science and Higher Education in 2013, Warsaw, Poland.

\section{References}

[1] Maczyński A., Positioning and Stabilization of the Load Position of Elevated Work Platforms, The University of Bielsko-Biala, Theses 14, 2005.

[2] Sochacki W., Dynamic Stability of Discrete-Continuous Mechanical Systems as Working Machine Models, Monographic series, No. 147, Czestochowa University of Technology, Częstochowa 2008.

[3] Cekus D., Posiadała B., Free vibrations of the system telescopic boom - hydraulic cylinder of crane radius change, Proceedings of the 21 Symposium: Vibration in Physical Systems, Poznań-Kiekrz 2004, 115-118.

[4] Sochacki W., Tomski L., Free and parametric vibration of the system: telescopic boom-hydraulic cylinder (changing the crane radius), The Archive of Mechanical Engineering 1999, 46, 257-271.

[5] Kirillov O.N., Seyranian A.O., The effect of small internal and external damping on the stability of distributed non-conservative systems, Journal of Applied Mathematics and Mechanics 2005, $69,529-552$.

[6] Gürgöze M., Doğruoğlu A.N., Aeren S., On the eigencharacteristics of a cantilevered visco-elastic beam carrying a tip mass and its representation by a spring-damper-mass system, Journal of Sound and Vibration 2007, 301, 420-426. 